\title{
Influence of Oxygen Concentration on Self-Compliance RRAM in Indium Oxide Film
}

\author{
Jyun-Bao Yang, Ting-Chang Chang, Senior Member, IEEE, Jheng-Jie Huang, Yu-Ting Chen, Hsueh-Chih Tseng, \\ Ann-Kuo Chu, Simon M. Sze, Ming-Jinn Tsai, Jin-Cheng Zheng, and Ding-Hua Bao
}

\begin{abstract}
This letter investigates various oxygen concentrations in indium oxide films which induce different resistance switching behaviors, including two self-compliance behaviors and a two-step set process. The accumulated oxygen ions produce an oxygen-rich indium oxide film, which can be considered as a variable series resistor after the forming process. Analyses indicate that the lower self-compliance current can be attributed to this larger variable series resistor from the additional oxygen ions. The more significant oxidation reaction decreases the current of the high resistance state. Hence, power consumption can be reduced effectively.
\end{abstract}

Index Terms-RRAM, nonvolatile resistance switching memory, indium oxide.

\section{INTRODUCTION}

$\mathbf{R}$ ECENTLY, with the advancement of portable electronic products, nonvolatile memories have attracted much attention [1]. With their scaling-down, conventional nonvolatile floating-gate memory has reached its physical limits [2]. Resistive random access memory (RRAM) is considered to replace conventional flash memory due to its advantageous properties of low operating power consumption [3], [4], good scalability, excellent retention, and fast operation speed [5], [6]. In this letter, we investigate the self-compliance behavior of indium oxide film with various oxygen concentrations in the $\mathrm{Pt} / \mathrm{InO}_{\mathrm{x}} / \mathrm{TiN}$ structure. It is notable that such RRAM devices with the indium oxide-based resistance switching (RS) layer including additional oxygen

Manuscript received April 24, 2014; revised May 15, 2014 and June 16, 2014; accepted June 27, 2014. Date of publication July 31, 2014; date of current version August 21, 2014. This work was supported by the National Science Council of Taiwan under Contract NSC-102-2120-M-110-001. The review of this letter was arranged by Editor M. Jurczak.

J.-B. Yang, Y.-T. Chen, and A.-K. Chu are with the Department of Photonics, National Sun Yat-sen University, Kaohsiung 804, Taiwan.

T.-C. Chang is with the Department of Photonics, Department of Physics, Center for Nanoscience and Nanotechnology, National Sun Yat-sen University, Kaohsiung 804, Taiwan, and also with the Advanced Optoelectronics Technology Center, National Cheng Kung University, Tainan 701, Taiwan (e-mail: tcchang@mail.phys.nsysu.edu.tw).

J.-J. Huang and H.-C. Tseng are with the Department of Physics, National Sun Yat-sen University, Kaohsiung 804, Taiwan.

S.-M. Sze is with the Department of Electronics Engineering, National Chiao Tung University, Hsinchu 300, Taiwan.

M.-J. Tsai is with the Electronics and Optoelectronics Research Laboratory, Industrial Technology Research Institute, Hsinchu 310, Taiwan.

J.-C. Zheng is with the Department of Physics, Xiamen University, Xiamen 361005, China.

D.-H. Bao is with the State Key Laboratory of Optoelectronic Materials and Technologies, School of Physics and Engineering, Sun Yat-sen University, Guangzhou 510275, China.

Color versions of one or more of the figures in this letter are available online at http://ieeexplore.ieee.org.

Digital Object Identifier 10.1109/LED.2014.2336676
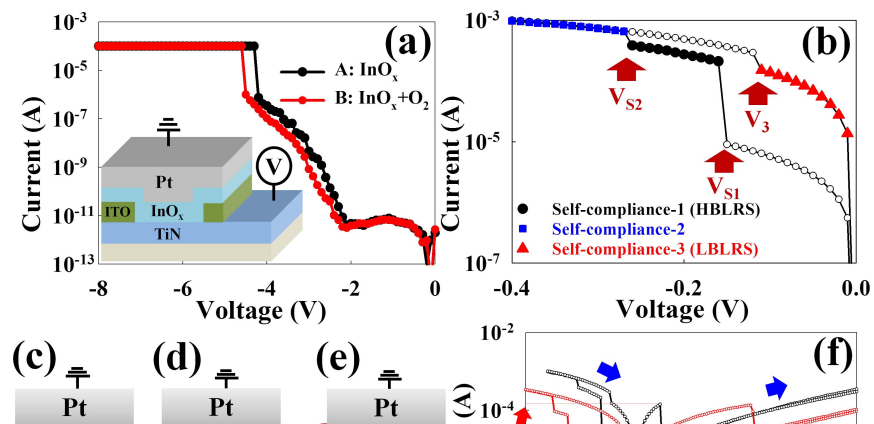

(d)

(e)
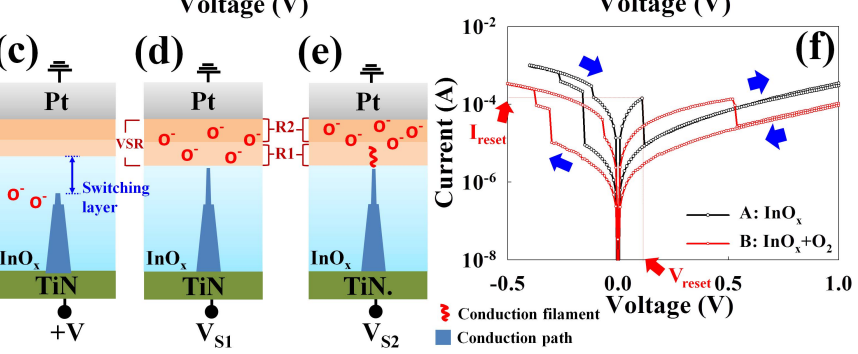

Fig. 1. (a) The forming process of samples A and B, and the inset shows structural diagram. (b) Bipolar resistance switching of sample A during the set process. The resistance switching mechanism during (c) reset process, (d) set process of self-compliance-1, and (e) set process of self-compliance-2.

(f) The current-voltage curve for samples A and B.

ions can produce the desirable properties of low power consumption and low operation current.

\section{EXPERIMENT}

The $200 \mathrm{~nm}$-thick low temperature silicon oxide (LTO) was deposited on $\mathrm{TiN} / \mathrm{SiO}_{2} / \mathrm{Si}$ structure and the cell area of $2 \times 2 \mu \mathrm{m}$ was defined by reactive ion etching the LTO. The $\mathrm{RS}$ layer of 20-nm indium oxide $\left(\mathrm{InO}_{\mathrm{x}}\right)$ was deposited on the TiN by RF sputtering an $\mathrm{In}_{2} \mathrm{O}_{3}$ target in $\mathrm{Ar}(30 \mathrm{sccm})$ ambience at room temperature, defined as sample A. Similarly, RS layer for sample B was also deposited on the substrate in $\operatorname{Ar}(30 \mathrm{sccm})$ and $\mathrm{O}_{2}(10 \mathrm{sccm})$ ambience at room temperature for comparison. Subsequently, the Pt top electrode was deposited on the $\mathrm{InO}_{\mathrm{x}}$ film by $\mathrm{DC}$ sputtering. During the measurement process, bias was applied on the TiN bottom electrode and the Pt top electrode was grounded, as shown in Fig. 1(a). All the RS characteristics were measured by an Agilent B1500 semiconductor parameter analyzer and a Cascade M150 probe station. In addition, the compositions of the indium oxide films were analyzed by X-ray photoelectron spectroscopy (XPS).

\section{RESUlt AND Discussion}

During the forming process, the conduction paths were constructed by applying $+8 \mathrm{~V}$ with a $100 \mu \mathrm{A}$ current compliance 
on both samples A and B, and the resistance state was switched to low resistance state (LRS) after the forming process. The forming voltage is defined as the voltage at the point where the current increases abruptly during the forming process. The forming voltage of sample A is lower than that of sample B, as shown in Fig. 1(a).

Figure 1(b) shows the current-voltage (I-V) curve of sample A during the set process, where the resistance state can be switched from high resistance state (HRS) to LRS with a negative voltage. The set voltage $\left(\mathrm{V}_{\text {set }}\right)$ is defined as the voltage at the point where the current increased abruptly. The conduction path of a metal-like filament is formed and large amount of oxygen ions drift to the Pt electrode due to the electric field direction during the forming process. Since the oxygen ions cannot react with Pt [7], the indium oxide layer with high concentration of oxygen forms a variable series resistor (VSR) between the conduction path and the Pt electrode, and the VSR region can be considered as an oxygen reservoir [8]-[10]. Because of the formation of the VSR, the self-compliance behavior is observed without an external current limit [11]. As the oxygen ions migrate to the conduction path due to the positive voltage, the oxygen ions react with indium atoms at the end conduction path and the resistance state is switched back to HRS (reset process), as shown in Fig. 1(c). The reset voltage $\left(\mathrm{V}_{\text {reset }}\right)$ is defined as the voltage at the point where the current starts its decrease and an insulator layer (switching layer) between the VSR and the end of the conduction path is formed, as shown in Fig. 1(c).

The two-step $\mathrm{V}_{\text {set }}$ as well as the two self-compliance behaviors are observed in the I-V curve during the set process, shown in Fig. 1(b). The current increases abruptly at $\mathrm{V}_{\mathrm{S} 1}$, indicating that the conduction path is re-formed and the selfcompliance-1 (higher bias LRS; HBLRS, ranging from $\mathrm{V}_{\mathrm{S} 1}$ to $V_{S 2}$ ) behavior is observed due to the formation of the VSR region, as shown in Fig. 1(d). Furthermore, as the bias continuously rises, the current abruptly increases at $\mathrm{V}_{\mathrm{S} 2}$ since the resistor of the indium oxide of a lower oxygen concentration (R1) in the VSR region undergoes soft breakdown by the higher electric field at end of the conduction path, and the formation of conduction filament (CF) is formed in VSR region, as shown in Fig. 1(e). Moreover, the self-compliance-2 behavior can be observed due to the resistor of a higher oxygen concentration (R2) in the VSR region. Subsequently, the current decreases abruptly and the self-compliance-3 (lower bias LRS; LBLRS, ranging from $\mathrm{V}_{3}$ to $0 \mathrm{~V}$ ) behavior occurs at $\mathrm{V}_{3}$ during the bias sweep back to $0 \mathrm{~V}$, because the $\mathrm{CF}$ is oxidized by its surrounding high-concentration oxygen ions, as well as the tip of conduction path also being oxidized by the high concentration of oxygen ions. In addition, although the formation of another parallel path at $\mathrm{V}_{\mathrm{S} 2}$ is possible, it is actually not likely. Since the rupture probability of two conduction path is the same, it is difficult to explain why the same conduction path always ruptures at $\mathrm{V}_{3}$ if there were parallel conduction paths. Hence, the RS mechanism in this manuscript is dominated by the single conduction path mode.

Figure 1(f) shows the typical bipolar RS behavior of samples A and B, including different self-compliance behaviors and the two-step $\mathrm{V}_{\text {set }}$. A comparison of $\mathrm{I}-\mathrm{V}$ curves
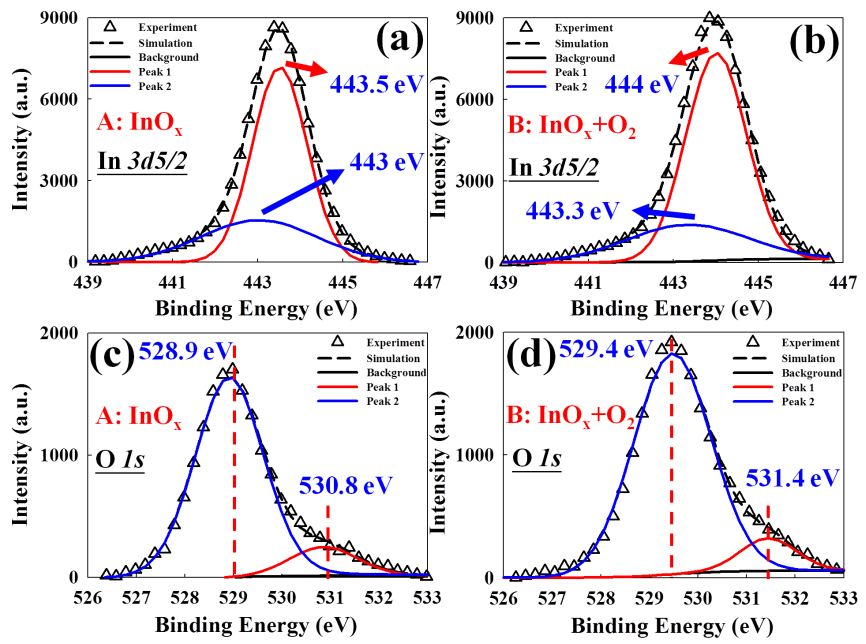

Fig. 2. XPS analysis of (a) In $3 d 5 / 2$ for sample A, (b) In $3 d 5 / 2$ for sample B, (c) $\mathrm{O} 1 \mathrm{~s}$ for sample $\mathrm{A}$, and (d) $\mathrm{O}$ 1s for sample $\mathrm{B}$.
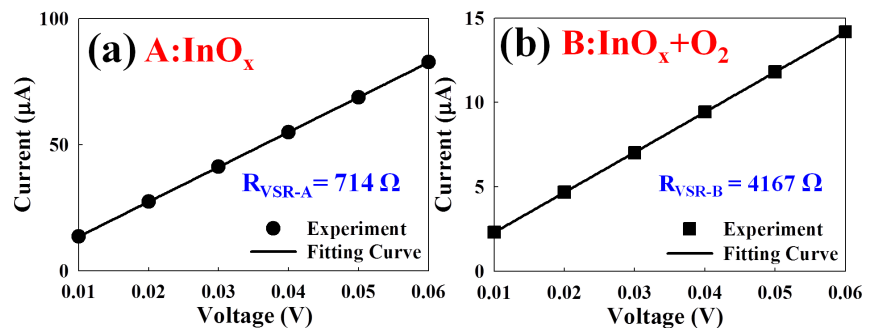

Fig. 3. The current transport mechanism of ohmic conduction (a) for sample A and (b) sample B in LBLRS.

between samples $A$ and $B$ shows that the $V_{\text {reset }}$ and $V_{\text {set }}$ for sample B is larger than those for sample A, and the operation current for sample B is lower than for sample A. They both exhibit a self-compliance behavior during the set process, with the self-compliance current in sample B being lower than in sample A.

Figure 2 shows the XPS analysis of the compositions of the indium oxide film. The spectrum peak of In $3 \mathrm{~d}_{5 / 2}$ and $\mathrm{O} 1 \mathrm{~s}$ are separated into two peaks in both samples A and B. In sample $\mathrm{A}$, one peak of In $3 \mathrm{~d}_{5 / 2}$ is that of In-O bonding $(443.5 \mathrm{eV})$, and the other peak is In-In bonding $(443 \mathrm{eV})$, as shown in Fig. 2(a). Furthermore, the Fig. 2(c) shows that the peaks of $\mathrm{O} 1 \mathrm{~s}$ in sample A are located at $528.9 \mathrm{eV}$ and $530.8 \mathrm{eV}$, representing In-O bonding and the oxygen ions in the oxygen deficient region [12], respectively.

In order to investigate the chemical states of the indium oxide film in samples A and B, the peak areas are calculated by the Lorenzian-Gaussian function. The composition ratio of $\mathrm{O}$ elements to In elements in sample A $(0.95: 1)$ is smaller than in sample B (1:1), which indicates that sample B with additional oxygen atoms can effectively enhance the oxygen concentration. Compared to sample A, the composition ratio of In-O to In-In bonding is increased in sample B. This result indicates that the RS layer with additional oxygen ions can reduce the quantity of defects and enhance the oxidation of In atoms.

In order to investigate the current transportation mechanism of LRS, the conduction mechanisms are analyzed [13]. Fig. 3(a) and (b) show that the conduction mechanisms of 
(a)
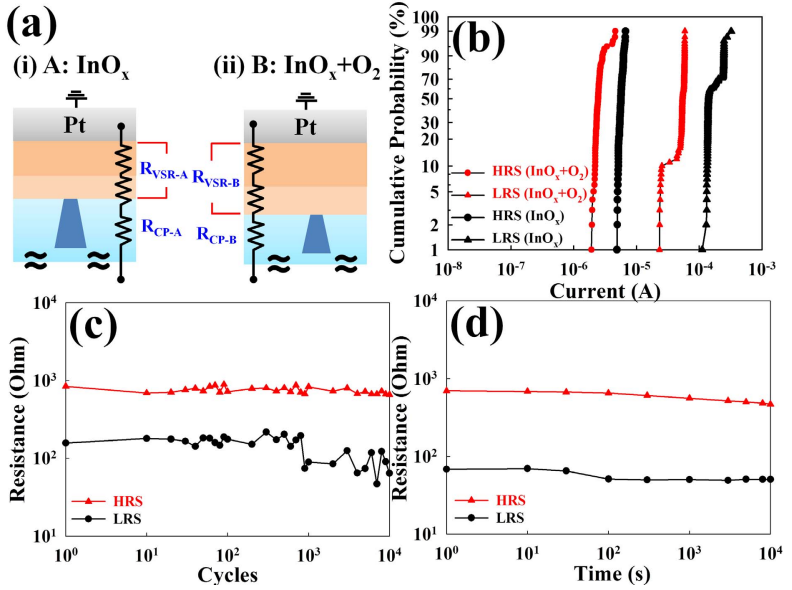

Fig. 4. (a) Schematic diagrams illustrating the formation of VSR and end of conduction path for samples A and B. (b) The cumulative distribution of the HRS and LRS in sample A and B. (c) Endurance for 104 cycles as tested by $\mathrm{AC}$ sweeping, and (d) the retention for $104 \mathrm{~s}$ at $85^{\circ} \mathrm{C}$.

LBLRS in both samples A and B follow Ohmic law. According to the resistance equation $(\mathrm{R}=\mathrm{V} / \mathrm{I})$, the resistance value can be obtained from the slope of Ohmic fitting. The R, V, and I are resistance value of VSR ( $\left.\mathrm{R}_{\mathrm{VSR}}\right)$, bias voltage, and current, respectively. The $R_{V S R}$ for sample A $\left(R_{V S R}-A=714 \Omega\right)$ is smaller than for sample B $\left(R_{V S R-B}=4167 \Omega\right)$.

Figure 4(a) shows the schematic diagram of the switching region for samples $\mathrm{A}$ and $\mathrm{B}$, which includes both the VSR and the end of conduction path, considered as the two resistors $\mathrm{R}_{\mathrm{VSR}}$ and $\mathrm{R}_{\mathrm{CP}}$ in series. Their different $\mathrm{R}_{\mathrm{VSR}}$ can be obtained from Fig. 3(a) and (b), whose result has demonstrated that $\mathrm{R}_{\mathrm{VSR}-\mathrm{B}}$ is larger than $\mathrm{R}_{\mathrm{VSR}-\mathrm{A}}$. The conduction path connects with a series VSR and the voltage crossing in VSR region should also be considered. According to simple voltage divider rule $\left(\mathrm{V}_{\mathrm{VSR}}=\left[\mathrm{R}_{\mathrm{VSR}} /\left(\mathrm{R}_{\mathrm{VSR}}+\mathrm{R}_{\mathrm{CP}}\right)\right] \mathrm{V}_{\mathrm{bias}}\right)$ the voltage crossing $\mathrm{R}_{\mathrm{VSR}-\mathrm{B}}$ is larger than $\mathrm{R}_{\mathrm{VSR}-\mathrm{A}}$ for the same bias conditions. Hence, compared to sample A, sample B requires a larger $\mathrm{V}_{\text {reset }}$ due to the higher voltage crossing $\mathrm{R}_{\mathrm{VSR}-\mathrm{B}}$ along the conduction path.

According to the electric power equation $\left(\mathrm{P}_{\text {reset }}=\mathrm{I}_{\text {reset }} \times\right.$ $\left.\mathrm{V}_{\text {reset }}\right), \mathrm{P}_{\text {reset }}, \mathrm{I}_{\text {reset }}$, and $\mathrm{V}_{\text {reset }}$ are reset power, reset current, and reset voltage, respectively. The driving power of sample B $(70.4 \mu \mathrm{W})$ is larger than that of sample $\mathrm{A}(15.6 \mu \mathrm{W})$ and the result indicates that the higher power induces a more significant oxidation reaction, and the thickness of the switching layer of sample B is larger that of sample A. Hence, the HRS current of sample B is lower than that of sample A. Besides a higher crossing voltage of $\mathrm{R}_{V S R-B}$, because of the thicker switching layer in sample $\mathrm{B}$, electrons require a higher energy to break the metal-oxide bond of the switching layer by a higher set bias. Hence, the $\mathrm{V}_{\text {set }}$ in sample $\mathrm{B}$ is larger than that sample A.

Figure 4(b) shows the obvious memory window read at $0.1 \mathrm{~V}$ during $1000 \mathrm{DC}$ operation cycles for sample A and B. The operation current for sample B is lower than that for sample A. Figure 4(c) shows the endurance of $10^{4}$ cycles for sample B by AC pulse operation. As applying a $-0.8 \mathrm{~V}$ with a $300 \mathrm{~ns}$ pulse width, the resistance state is switched from HRS to LRS. Conversely, the resistance state is switched back to HRS by a $+0.8 \mathrm{~V}$ operation voltage with a $600 \mathrm{~ns}$ pulse width. The result indicates that the device can be switched at least 104 cycles and still retain approximate 1 order of magnitude of on/off ratio. After the $10^{4}$ cycles of endurance test, the HRS and LRS retain well without degradation longer than $10^{4} \mathrm{~s}$ at $85^{\circ} \mathrm{C}$, as shown in Fig. 4(d).

\section{CONCLUSION}

In summary, in order to investigate the influence of different oxygen concentrations in an indium oxide-based RS layer, the indium oxide film is deposited by sputtering in ambient Ar (sample A) and in $\mathrm{Ar} / \mathrm{O}_{2}$ (sample B). According to the presented analyses of the electric power, a significant reset behavior lowers the HRS current. The $V_{\text {set }}$ in sample $B$ is larger than that of sample A due to the thicker switching layer and higher $\mathrm{R}_{\mathrm{VSR}-\mathrm{B}}$. According to the resistance value from VSR analysis, the $V_{\text {reset }}$ in sample $B$ is larger than in sample A, since a higher resistance value for the VSR forms in B. Furthermore, this higher resistance value can decrease the selfcompliance current and achieve lower power consumption due to its lower operation current.

\section{ACKNOWLEDGMENT}

This work was performed at National Science Council Core Facilities Laboratory for Nano-Science and Nano-Technology in Kaohsiung-Pingtung area, NSYSU Center for Nanoscience and Nanotechnology.

\section{REFERENCES}

[1] A. Campera and G. Iannaccone, "Modelling and simulation of charging and discharging processes in nanocrystal flash memories during program and erase operations," Solid-State Electron., vol. 49, p. 1745, Sep. 2005.

[2] T.-C. Chang et al., "Developments in nanocrystal memory," Mater. Today, vol. 14, no. 12, pp. 608-615, 2011.

[3] A. Sawa, "Resistive switching in transition metal oxides," Mater. Today, vol. 11 , no. 6, pp. 28-36, 2008.

[4] Y.-E. Syu et al., "Redox reaction switching mechanism in RRAM device with $\mathrm{Pt} / \mathrm{CoSiO}{ }_{X} / \mathrm{TiN}$ structure," IEEE Electron Device Lett., vol. 32, no. 4, pp. 545-547, Apr. 2011

[5] M.-C. Chen et al., "Influence of electrode material on the resistive memory switching property of indium gallium zinc oxide thin films," Appl. Phys. Lett., vol. 96, no. 26, pp. 262110-1-262110-3, Jun. 2011.

[6] Y.-E. Syu et al., "Atomic-level quantized reaction of $\mathrm{HfO}_{x}$ memristor," Appl. Phys. Lett., vol. 120, no. 17, pp. 172903-1-172903-4, Apr. 2013.

[7] L. Goux et al., "Evidences of oxygen-mediated resistive-switching mechanism in TiN/HfO 2 /Pt cells," Appl. Phys. Lett., vol. 97, no. 24, pp. 243509-1-243509-3, Dec. 2010.

[8] Y.-S. Chen et al., "Novel defects-trapping $\mathrm{TaO}_{x} / \mathrm{HfP}_{x}$ RRAM with reliable self-compliance, high nonlinearity, and ultra-low current," IEEE Electron Device Lett., vol. 35, no. 2, pp. 202-204, Feb. 2014.

[9] M. Predanocy et al., "Study of optical and electrical properties of sputtered indium oxide films," in Proc. 8th Int. Conf. Adv. Semicond. Devices Microsyst. (ASDAM), Oct. 2010, pp. 297-300.

[10] W. Banerjee et al., "Formation polarity dependent improved resistive switching memory characteristics using nanoscale $(1.3 \mathrm{~nm})$ core-shell $\mathrm{IrO}_{x}$ nano-dots," Nanoscale Res. Lett., vol. 7, p. 194, Mar. 2012.

[11] C. B. Lee et al., "Highly uniform switching of tantalum embedded amorphous oxide using self-compliance bipolar resistive switching," IEEE Electron Device Lett., vol. 32, no. 3, pp. 399-401, Mar. 2011.

[12] A. Younis, D. Chu, and S. Li, "Tuneable resistive switching characteristics of $\mathrm{In}_{2} \mathrm{O}_{3}$ nanorods array via Co doping," RSC Adv., vol. 3, pp. 13422-13428, May 2013.

[13] S. M. Sze and K. K. Ng, Physics of Semiconductor Devices, 3rd ed. Hoboken, NJ, USA: Wiley, 2006. 